\title{
Effects of femtosecond laser-assisted cataract surgery on dry eye
}

\author{
DEWANG SHAO ${ }^{1}$, XIAOQUAN ZHU $^{1}$, WEI SUN ${ }^{1}$, PENG CHENG $^{1}$, WEI CHEN ${ }^{2}$ and HUA WANG ${ }^{2}$ \\ ${ }^{1}$ Department of Ophthalmology, Air Force Aviation Medicine Research Institute Affiliation Hospital; \\ ${ }^{2}$ Department of Ophthalmology, Air Force General Hospital, Beijing 100089, P.R. China
}

Received April 9, 2018; Accepted October 9, 2018

DOI: $10.3892 / \mathrm{etm} .2018 .6862$

\begin{abstract}
The aim of this study was to investigate the effect of femtosecond laser-assisted cataract surgery (FLACS) on tear film and ocular surface function. Patients with age-related cataract who underwent phacoemulsification (phaco) in Air Force Aviation Medicine Research Institute Affiliation Hospital from January 2016 to December 2016 were randomly divided into two groups. Patients in experimental group $(n=123,150$ eyes) received FLACS, while patients in phaco group $(n=110,150$ eyes) underwent conventional coaxial micro-incision phaco and were implanted with foldable intraocular lens (IOL). Our results showed that there were no statistically significant differences in general conditions between the two groups. Ocular surface disease index (OSDI) and corneal fluorescein staining (CFS) scores of patients in both groups after operation were significantly increased compared with those before operation, but breakup time (BUT) and Schirmer's I test scores decreased significantly at 1 week after operation compared with those before operation and basically returned to preoperative baseline levels at 3 months after operation. CFS scores and OSDI in the FLACS group at 1 day and 1 week after operation were increased more significantly than those in the phaco group, but there were no statistically significant differences at 3 months after operation. The above results showed that the effect on ocular surface function in the FLACS group after operation was greater than that in the phaco group, and the dry eye symptoms in the FLACS group were more obvious after operation. Both FLACS and conventional phaco have certain effects on the ocular surface function of patients, and there are statistically significant differences only in the early stage. FLACS has greater effects on CFS and dry eye symptoms.
\end{abstract}

Correspondence to: Dr Dewang Shao, Department of Ophthalmology, Air Force Aviation Medicine Research Institute Affiliation Hospital, 15 Changyungong, Beijing 100089, P.R. China E-mail: sgqye973@163.com

Key words: femtosecond laser, cataract surgery, ocular surface, dry eye

\section{Introduction}

Femtosecond laser is a kind of ultra-short pulse laser with high instantaneous power, small focus size, strong penetrability and high precision. It has been successfully applied in refractive surgery and corneal transplantation, in recent years $(1,2)$. Since 2010, femtosecond laser has been used in cataract surgery, which, assisted by a high-definition ocular anterior segment imaging system, can complete the precise anterior capsule circular incision, individualized surgical incision, corneal release astigmatism correction and safe pre-chopping, greatly improving the safety of cataract surgery and the predictability of postoperative outcomes $(3,4)$. The success of this technique indicates that the cataract surgery is quicker and safer, and the effect after intraocular lens (IOL) implantation is more satisfactory.

A large number of studies have shown that some patients have dry eye symptoms after receiving phacoemulsification (phaco), such as dryness, foreign body sensation, burning sensation and blurred vision (5-7). Microscopic ocular surface damage is a pathogenic factor of dry eyes after cataract surgery, which can cause ocular discomfort. This research aimed to study the dry eyes of patients before and after femtosecond laser-assisted cataract surgery (FLACS) and compare the effects of FLACS and conventional cataract surgery on the ocular surface function of patients.

\section{Patients and methods}

Subjects of study. Patients with age-related cataract who underwent phaco in Air Force Aviation Medicine Research Institute Affiliation Hospital (Beijing, China) from January 2016 to December 2016 were randomly divided into two groups. Patients in experimental group ( $n=123,150$ eyes) received FLACS, while patients in phaco group $(n=110$, 150 eyes) underwent conventional coaxial micro-incision phaco and were implanted with foldable IOL. In the FLACS group, there were 60 males (67 eyes) and 63 females ( 83 eyes) aged $54-78$ years $(65.74 \pm 11.80$ years on average). In the phaco group, there were 51 males (62 eyes) and 59 females (88 eyes) aged 56-81 years $(69.05 \pm 12.61$ years on average). There were no statistically significant differences in the sex ratio, average age and preoperative routine eye examination results, between the two groups (Table I). All patients underwent preoperative routine eye examinations (including uncorrected visual acuity, best corrected visual acuity, intraocular pressure 
measurement, slit-lamp examination, ocular A ultrasound, ocular B ultrasound and IOL power measurement) and other relevant auxiliary examinations (including blood routine, stool routine, urine routine, hepatic and renal function tests, blood glucose test, eight-item detection before blood transfusion, electrocardiogram and anterioposterior and lateral chest film) in the Department of Ophthalmology.

None of the patients had dry eyes. The diagnostic criteria for dry eyes were based on the Japanese Diagnostic Criteria for Dry Eye (8), namely the following three criteria: Schirmer's I test (SIt) $\leq 10 \mathrm{~mm}$, breakup time (BUT) $\leq 5 \mathrm{sec}$; corneal fluorescein staining $(\mathrm{CFS}) \geq 1$; symptoms, such as dryness, foreign body sensation and burning sensation.

All patients suffered from age-related cataract without active inflammation, such as conjunctivitis and keratitis, within 3 months before operation. No eye-drop irritation symptoms occurred locally. Patients did not wear contact lenses, and had no history of ophthalmic surgery, eye trauma, eyelid varus-valgus, incomplete closure, dacryocystitis, uveitis, glaucoma, fundus diseases or other eye diseases; they were not complicated by other systemic metabolic and immune diseases affecting tear secretion; they did not use eye drops or oral glucocorticoid hormones affecting the tear film function locally within 6 months. Those patients with bilateral cataract received surgery at an interval $>1$ month, so as to avoid dry eye symptoms in both eyes and cross influence of signs. All patients were followed up for $>3$ months after operation, and all objects of study were informed of the study and signed the informed consent. This study was approved by the Ethics Committee of Air Force Aviation Medicine Research Institute Affiliation Hospital.

Surgical methods. FLACS: under surface anesthesia (Benoxil; Santen Pharmaceutical Co., Ltd., Beijing, China), the patients lay on the operating bed, and the femtosecond laser was used to make the main corneal incision $(2.3 \mathrm{~mm})$ and side incision $(1.0 \mathrm{~mm})$, perform capsulorhexis $(4.6 \mathrm{~mm} / 4.8 \mathrm{~mm})$ and chop nucleus (energy parameter, $10 \mu \mathrm{J})$ (LenSx; Alcon Laboratories, Inc., Fort Worth, TX, USA). The corneal incision was bluntly separated using an iris restorer into the anterior chamber, the anterior chamber was filled with viscoelastic agent, and the anterior capsule was removed and separated using water. After the energy was adjusted to $100 \%$ and the suction to $450 \mathrm{mmHg}$ $(1 \mathrm{mmHg}=0.133 \mathrm{kPa})$, phaco was performed (INFINITI phaco instrument; Alcon Laboratories, Inc.), residual cortices were sucked in I/A mode, and IOL was implanted into a capsular bag and adjusted to the normal position. Then viscoelastic agent was injected, and the puncture site was hydrated, forming the anterior chamber. Tobramycin and Dexamethasone eye ointments (Yangtze River Pharmaceutical Group Co., Ltd., Taizhou, China) were used and the operative eyes were bandaged.

Phaco: under surface anesthesia (Benoxil; Santen Pharmaceutical Co., Ltd.), a transparent corneal incision was made at $2.2 \mathrm{~mm}$ above the temple or nose, and an auxiliary corneal incision was made at $2.00 \mathrm{~cm}$ on the lateral side. After injection of viscoelastic agent into the anterior chamber, continuous circular capsulorhexis, hydrodissection and in situ chopping method in the capsular bag, phaco was performed for lens nucleus (ultrasound energy of $2-30 \%, 8 \%$ on average). After removal of cortices, the capsular bag was implanted with a
Table I. Demographics and baseline characteristics of patients undergoing cataract surgery.

\begin{tabular}{lccc}
\hline Parameter & $\begin{array}{c}\text { FLACS group } \\
(\mathrm{n}=150)\end{array}$ & $\begin{array}{c}\text { Phaco group } \\
(\mathrm{n}=150)\end{array}$ & P-value \\
\hline Age (years) & $65.74 \pm 11.80$ & $69.05 \pm 12.61$ & 0.848 \\
Sex (n, \%) & & & \\
Male & $67(44.7)$ & $62(41.3)$ & 0.656 \\
Female & $83(55.3)$ & $88(58.7)$ & \\
Mean IOP (mmHg) & $12.50 \pm 4.69$ & $13.86 \pm 3.07$ & 0.809 \\
AL (mm) & $24.19 \pm 1.34$ & $23.87 \pm 1.06$ & 0.852 \\
ACD (mm) & $2.85 \pm 0.33$ & $2.79 \pm 0.42$ & 0.911 \\
Mean K (D) & $44.13 \pm 1.95$ & $43.80 \pm 1.76$ & 0.900 \\
\hline
\end{tabular}

FLACS, femtosecond laser-assisted cataract surgery; phaco, phacoemulsification; IOP, intraocular pressure; AL, axial length; $A C D$, anterior chamber depth; K, keratometry; D, diopters.

foldable IOL, and Tobradex eye ointment (Yangtze River Pharmaceutical Group Co., Ltd.) was used in the conjunctival sac after operation.

All operations were performed by one experienced cataract specialist. The same equipment and instruments were used during operation, and Tobramycin, Dexamethasone and Pranoprofen eye drops (Yangtze River Pharmaceutical Group Co., Ltd.) were used after operation for 4 weeks (4 times/day in the 1 st week, and then decreased progressively by 1 day every week).

Examination methods. At 1 day before operation, and at 1 day, 1 week, 1 month and 3 months after operation, the ocular surface disease index (OSDI), BUT and tear meniscus height $(\mathrm{TMH})$ were evaluated, and the SIt and CFS were performed; the observation data were recorded.

OSDI was used for dry eye symptoms according to a previous study (9). The questionnaire included 12 major questions about eye discomfort (questions 1-3), visual function (questions 4-9) and environmental triggers (questions 10-12). The score for each question was based primarily on the duration of symptoms in patients: 4 points, all the time; 3 points, most of the time; 2 points, half of the time; 1 point, a short time (occasionally); 0 point, never. Patients did not need to answer all 12 questions. The total score of OSDI was 0-100. Total score of OSDI = total scores for all questions $\mathrm{x} 25 /$ the number of questions answered.

In SIt, standard $5 \mathrm{~mm}$ x $35 \mathrm{~mm}$ Whatman filter paper (YZB/0360-2004; Tianjin Jingming New Technological Development Co., Ltd., Tianjin, China) was used; patients sat with their backs to the light in a dark room; one end of the filter paper was folded and placed at $1 / 3$ junction of lower-eyelid conjunctival sac, and the other end naturally drooped; patients closed their eyes gently, and the filter paper was removed after $5 \mathrm{~min}$. The length of tears soaking the filter paper was measured with a ruler in millimeters.

In CFS, the cornea was observed under the blue light of slit lamp. The cornea was divided into four quadrants (superior 
Table II. OSDI before and after cataract surgery in the operated eyes of the study patients.

\begin{tabular}{lccc}
\hline Time & FLACS group & Phaco group & P-value \\
\hline Baseline & $0.45 \pm 0.23$ & $0.47 \pm 0.39$ & 0.965 \\
Postoperative 1 day & $5.34 \pm 0.49$ & $4.04 \pm 0.33$ & 0.028 \\
Postoperative 1 week & $4.99 \pm 0.53$ & $3.47 \pm 0.55$ & 0.048 \\
Postoperative 1 month & $2.23 \pm 0.66$ & $1.83 \pm 0.71$ & 0.680 \\
Postoperative 3 months & $0.59 \pm 0.30$ & $0.51 \pm 0.39$ & 0.394 \\
\hline
\end{tabular}

OSDI, ocular surface disease index; FLACS, femtosecond laser-assisted cataract surgery; phaco, phacoemulsification.

Table III. SIt values before and after cataract surgery in the operated eyes of the study patients $(\mathrm{mm})$.

\begin{tabular}{lrrr}
\hline Time & FLACS group & Phaco group & P-value \\
\hline Baseline & $10.92 \pm 4.13$ & $9.37 \pm 3.98$ & 0.829 \\
Postoperative 1 day & $11.27 \pm 4.87$ & $10.68 \pm 3.73$ & 0.923 \\
Postoperative 1 week & $7.55 \pm 3.69$ & $7.20 \pm 3.29$ & 0.944 \\
Postoperative 1 month & $8.83 \pm 2.58$ & $8.04 \pm 2.73$ & 0.834 \\
Postoperative 3 months & $11.15 \pm 4.99$ & $10.08 \pm 5.35$ & 0.884
\end{tabular}

SIt, Schirmer's I test; FLACS, femtosecond laser-assisted cataract surgery; phaco, phacoemulsification.

temporal, inferior temporal, superior nasal and inferior nasal) to observe the presence of fluorescein staining in each quadrant. No staining, 0 point; dotted and small pieces of staining, 1 and 2 points; block staining, 3 points; a total of 0-12 points.

In non-invasive tear-film assessment, a newly developed corneal topographer (Oculus Optikgeräte $\mathrm{GmbH}$, Wetzlar, Germany) was used to measure the TMH, and non-invasive tear film BUT according to previous studies (10-12), including the first and the average BUT. The procedures were conducted as per the protocol in previous literature.

All examinations were performed by the same experienced physician in the same quiet examination room.

Statistical analysis. Statistical Product and Service Solutions (SPSS) 13.0 software (SPSS, Inc., Chicago, IL, USA) was used for statistical processing. $\mathrm{W}$ test showed that the test indexes in this study were normally distributed, continuous variables were presented as mean \pm standard deviation (SD), and Levene's test showed the homogeneity of variance. One-way repeated measurement data analysis of variance was used for the comparison of test index between FLACS and phaco group at different time-points before and after operation, and least significant difference (LSD) t-test was used for the pairwise comparison. Independent-samples t-test was used for the comparison of the sample mean between two groups at the same time-point, and Chi-square test was used for the categorical data. $\mathrm{P}<0.05$ was considered to indicate a statistically significant difference.
Table IV. CFS scores before and after cataract surgery in the operated eyes of the study patients.

\begin{tabular}{lccc}
\hline Time & FLACS group & Phaco group & P-value \\
\hline Baseline & $0.46 \pm 0.20$ & $0.38 \pm 0.22$ & 0.788 \\
Postoperative 1 day & $2.34 \pm 0.31$ & $1.22 \pm 0.28$ & 0.008 \\
Postoperative 1 week & $1.88 \pm 0.29$ & $1.02 \pm 0.21$ & 0.017 \\
Postoperative 1 month & $0.97 \pm 0.20$ & $0.48 \pm 0.14$ & 0.046 \\
Postoperative 3 months & $0.51 \pm 0.39$ & $0.46 \pm 0.35$ & 0.775 \\
\hline
\end{tabular}

CFS, corneal fluorescein staining; FLACS, femtosecond laser-assisted cataract surgery; phaco, phacoemulsification.

\section{Results}

OSDI. Compared with that before operation, OSDI in both groups at 1 day and 1 week after operation was significantly increased, and the differences were statistically significant $(\mathrm{P}<0.05)$. The main symptom in patients was foreign body sensation. There was a statistically significant difference in OSDI in the FLACS group at 1 month after operation compared with that before operation $(\mathrm{P}=0.011)$, but there was no statistically significant difference in the phaco group compared with that before operation $(\mathrm{P}=0.094)$. There were no statistically significant differences in OSDI in both groups at 3 months after operation compared with that before operation $(\mathrm{P}>0.05)$. Compared with the phaco group, OSDI in the FLACS group at 1 day and 1 week after operation was increased, and the differences were statistically significant $(\mathrm{P}<0.05)$; the foreign body sensation and discomfort were more severe than those in the phaco group; there were no statistically significant differences between the two groups at 1 month and 3 months after operation $(\mathrm{P}>0.05)$ (Table II).

SIt value. Before operation, SIt values in the FLACS and the phaco group were $10.92 \pm 4.13$ and $9.37 \pm 3.98 \mathrm{~mm}$, respectively. SIt values in both groups were significantly decreased at 1 week after operation (7.55 \pm 3.69 and $7.20 \pm 3.29 \mathrm{~mm}$, respectively). SIt values increased at 1-3 months after operation and returned to preoperative levels at 3 months after operation (Table III). There was no statistically significant difference in SIt between the two groups $(\mathrm{P}>0.05)$.

CFS score. CFS scores in both groups after operation were significantly increased compared with those before operation, and the differences were statistically significant $(\mathrm{P}<0.05)$. CFS scores were the highest at 1 day after operation, gradually decreased afterwards, and returned to preoperative levels at 3 months after operation. CFS scores in the FLACS group at 1 day, 1 week and 1 month after operation were higher than those in the phaco group, and the differences were statistically significant ( $\mathrm{P}=0.008,0.017$ and 0.046, respectively) (Table IV).

First and average BUT. In this study, the first and average BUT in both groups at 1 week after operation were significantly shortened compared with those before operation, and the differences were statistically significant $(\mathrm{P}<0.05)$; indicating 

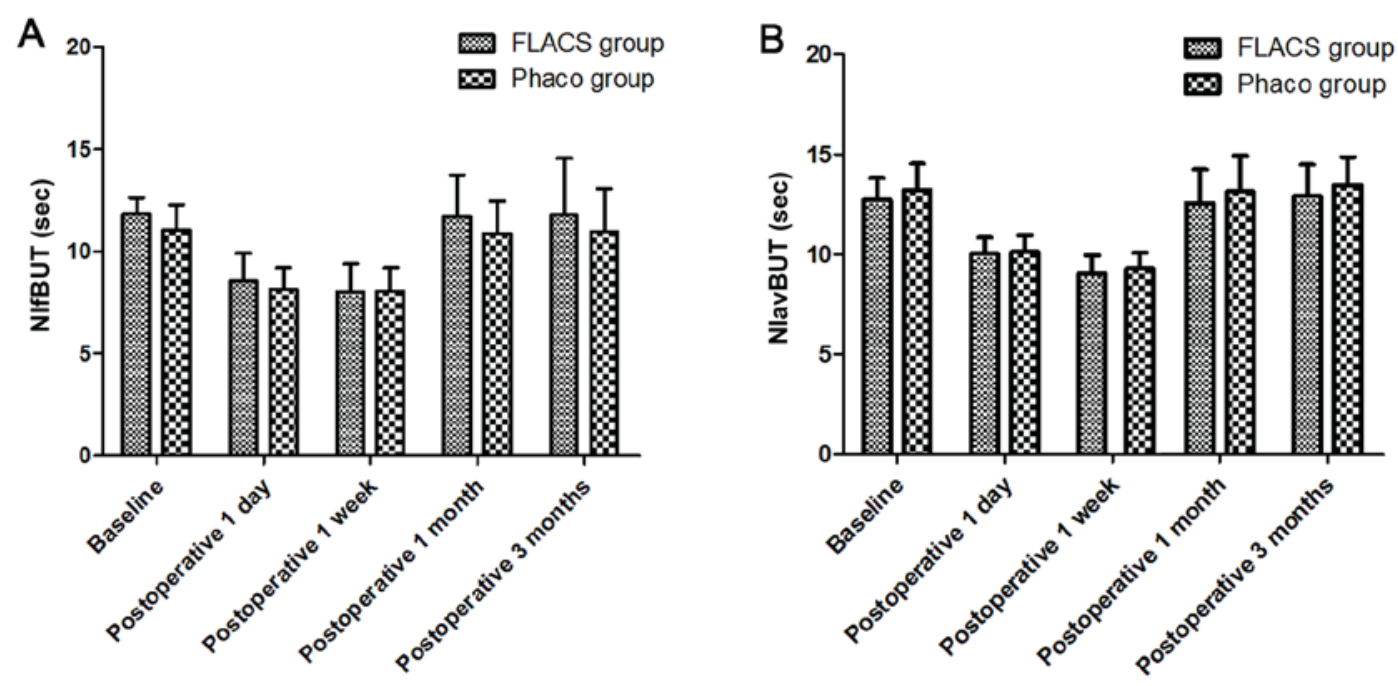

Figure 1. Changes in (A) NIfBUT and (B) NIavBUT before and after cataract surgery in the operated eyes of the study patients in the two groups. NIfBUT, non-invasive first breakup time; NIavBUT, non-invasive average breakup time; FLACS, femtosecond laser-assisted cataract surgery; phaco, phacoemulsification.

Table V. NIfBUT and NIavBUT values before and after cataract surgery in the operated eyes of the study patients (sec).

\begin{tabular}{lrrr}
\hline Parameters & \multicolumn{1}{c}{$\begin{array}{c}\text { FLACS } \\
\text { group }\end{array}$} & \multicolumn{1}{c}{$\begin{array}{c}\text { Phaco } \\
\text { group }\end{array}$} & P-value \\
\hline NIfBUT & & & \\
Baseline & $11.83 \pm 0.79$ & $11.04 \pm 1.24$ & 0.592 \\
Postoperative 1 day & $8.54 \pm 1.36$ & $8.16 \pm 0.04$ & 0.825 \\
Postoperative 1 week & $8.01 \pm 1.38$ & $8.07 \pm 1.13$ & 0.973 \\
Postoperative 1 month & $11.68 \pm 2.05$ & $10.89 \pm 1.57$ & 0.760 \\
Postoperative 3 months & $11.80 \pm 2.77$ & $10.97 \pm 2.11$ & 0.812 \\
NIavBUT & & & \\
Baseline & $12.71 \pm 1.09$ & $13.21 \pm 1.33$ & 0.771 \\
Postoperative 1 day & $10.01 \pm 0.81$ & $10.12 \pm 0.82$ & 0.924 \\
Postoperative 1 week & $9.03 \pm 0.92$ & $9.34 \pm 0.88$ & 0.808 \\
Postoperative 1 month & $12.56 \pm 1.68$ & $13.15 \pm 1.77$ & 0.809 \\
Postoperative 3 months & $12.90 \pm 1.59$ & $13.44 \pm 1.43$ & 0.801 \\
\hline
\end{tabular}

NIfBUT, non-invasive first breakup time; NIavBUT, non-invasive average breakup time; FLACS, femtosecond laser-assisted cataract surgery; phaco, phacoemulsification.

that the stability of tear film is decreased after operation. BUT returned to the preoperative level at 3 months after operation, and there were no statistically significant differences at 1 and 3 months after operation compared with those before operation $(\mathrm{P}>0.05)$. First and average BUT had no statistically significant differences between the two groups (Fig. 1; Table V).

$T M H$. TMH in both groups was slightly increased at 1 day after operation; it was decreased at 1 week after operation compared with that before operation, and the difference was statistically significant $(\mathrm{P}<0.05)$. At 1 and 3 months after operation, TMH basically returned to the preoperative levels, and the differences were not statistically significant $(\mathrm{P}>0.05)$.
Table VI. TMH values before and after cataract surgery in the operated eyes of the study patients (mm).

Time

FLACS group Phaco group P-value

\begin{tabular}{llll}
\hline Baseline & $0.37 \pm 0.09$ & $0.35 \pm 0.08$ & 0.868 \\
Postoperative 1 day & $0.41 \pm 0.13$ & $0.44 \pm 0.11$ & 0.860 \\
Postoperative 1 week & $0.22 \pm 0.07$ & $0.20 \pm 0.06$ & 0.828 \\
Postoperative 1 month & $0.32 \pm 0.05$ & $0.30 \pm 0.06$ & 0.798 \\
Postoperative 3 months & $0.36 \pm 0.07$ & $0.37 \pm 0.06$ & 0.925
\end{tabular}

TMH, tear meniscus height; FLACS, femtosecond laser-assisted cataract surgery; phaco, phacoemulsification.

There was no statistically significant difference in $\mathrm{TMH}$ between the two groups (Table VI).

\section{Discussion}

Tear film is a basis for maintaining the normal structure and function of ocular surface epithelium, which can moisten and protect the corneal and conjunctival epithelium. The stability of normal ocular surface tear film depends on the normal quality and quantity of tear film lipid layer, aqueous layer and mucinous layer and normal tear kinetics. When a variety of reasons lead to tear film instability and abnormal tear secretion, ocular surface changes can be caused, leading to dry eyes. Studies have found that all ophthalmic surgeries can cause tear film instability and postoperative dry eye syndrome $(12,13)$. Whether tear film and ocular surface will be affected after FLACS, as a kind of surgery with small trauma and fast recovery, is rarely studied.

The results of this study showed that the first BUT, average BUT and TMH in both groups were shortened significantly, and OSDI and CFS scores of dry eyes were significantly increased at 1 day and 1 week after operation compared with those before operation, which may be associated with the direct damage 
of phaco combined with IOL implantation to ocular surface. Currently, scholars world-wide consider that the reasons why phaco combined with IOL implantation reduces the stability of tear film are the following: frequent application of eye drops for preoperative local surface anesthesia; mechanical damage to ocular surface epithelium caused by preoperative irrigation and improper surgical skills; peripheral nerve injury at the incision and decreased local corneal sensation caused by transparent corneal incision (14); damage of preservatives in hormonal eye drops and local eye drops used after surgery to the ocular surface epithelial tissues $(15,16)$; postoperative exposure of ocular surface to hard light and repeated washing with lavage fluid, and damage to ocular surface goblet cell density and shortening of BUT (17); local and surrounding tissue edema, and patients' emphasis on the surgery and psychological factors.

It was found that patient usually have most of the chief complaints at 1 day after operation when the bandage was opened. The reason may be that the tear component in an eye-closed state after bandage is different from that when the eyes are opened. When eyes are opened in the daytime, the tear film is in a dynamic balance, promoting the secretion of fresh tears and removing tears. But the removal rate of tears becomes slow when eyes are closed at night. Besides, tears contain a large amount of immunoglobulin A ( $\operatorname{Ig} \mathrm{A})$, albumin concentration is also increased, plasminogen activators convert $\mathrm{C}_{3}$ into $\mathrm{C}_{3 \mathrm{c}}$, and a large number of neutrophils are aggregated, which is known as subclinical inflammation, and such a change is the most obvious at 3-5 $\mathrm{h}$ after the eyes are closed $(18,19)$. In the present study, the patients' eyes were bandaged for a long time, and the stress of operative wound and trauma made the epithelial cells secrete a large number of inflammatory factors accumulating in the tears, aggravating the inflammatory response. Inflammation leads to ocular surface epithelial damage, decreased stability of tear film, changes in tear component, and more main complaints of discomfort.

Each index in both groups returned to preoperative level at 90 days after operation, and the possible reasons are the following: the local eye drops applied after operation have a certain anti-inflammatory effect; when inflammation occurs in patients receiving phaco combined with IOL implantation, abundant lymphocyte infiltration can be found on ocular surface in patients with dry eyes, and ocular surface epithelial cells will produce a large number of pro-inflammatory cytokines, which will induce the phospholipase A2 in corneal and conjunctival epithelium to catalyze the phopholipid layer and convert it into arachidonic acid; furthermore, cyclooxygenase (COX) will decompose arachidonic acid to produce prostaglandins and thromboxane, and these products will further induce the inflammatory response and aggravate dry eyes. Non-steroidal anti-inflammatory drugs (NSAIDs) can inhibit the occurrence of inflammation through inhibiting the decomposition of arachidonic acid via COX, so they have a certain repair effect on the ocular surface. Secondly, the operative incision is small and it is located at the 11 o'clock direction of cornea; corneal nerves controlling the corneal sensation are mainly at 3 and 9 o'clock directions, and only a few nerve fibers are cut off during operation. Some studies have also shown that nerve growth factors are released onto the corneal epithelial regenerating axon at 25 days after operation, producing new nerve cells and healing the cornea (20), which also explains why dry eye symptoms are improved in early stage.

It was found in our study that CFS scores and OSDI in the FLACS group at 1 day and 1 week after operation were increased more significantly than those in the phaco group, but there were no statistically significant differences at 3 months after operation, indicating that the effect on ocular surface function of patients in the FLACS group was larger than that in the phaco group, and the dry eye symptoms after operation were also more obvious. This may be related to the peri-conjunctival injury caused by the suction ring used in femtosecond laser, which is similar to the pathological changes in dry eyes caused by laser-assisted in situ keratomileusis (LASIK) $(21,22)$. In addition, the irregular corneal surface damage due to surgical incision made by Sinskey hook is also an important cause, which is consistent with the corneal epithelial infiltration and fluorescence staining around the surgical incision that we observed in most patients after operation.

There were still many shortcomings in this study. The sample size was too small. In order to study the effect of FLACS on ocular surface function, more subjects of clinical study should be enrolled for large-sample data analysis; there are many influencing factors of ocular surface dysfunction after FLACS, such as the preoperative and postoperative application of local eye drops, intraoperative application of local surface anesthetics and operation time; but these factors affecting the experimental results were not taken into account in this study, so the influences of these factors should be fully lowered to reduce the error and deviation in subsequent studies; moreover, patients were followed up for a very short time, so the postoperative follow-up time should be prolonged in a future research to explore the long-term impact of FLACS on ocular surface.

In conclusion, both FLACS and conventional phaco can reduce the tear film stability of patients in a short term, thus affecting the ocular surface function. The effects of FLACS on CFS and dry eye symptoms are greater than those of conventional phaco.

\section{Acknowledgements}

Not applicable.

\section{Funding}

No funding was received.

\section{Availability of data and materials}

All data generated or analyzed during this study are included in this published article.

\section{Authors' contributions}

DS and HW designed the study and performed the experiments; DS, XZ, WS and PC collected the data; WC and HW analyzed the data; DS prepared the manuscript. All authors read and approved the final manuscript. 


\section{Ethics approval and consent to participate}

This study was approved by the Ethics Committee of Air Force Aviation Medicine Research Institute Affiliation Hospital (Beijing, China). Patients or their guardians provided written informed consent for publication.

\section{Patient consent for publication}

Not applicable.

\section{Competing interests}

The authors declare that they have no competing interests.

\section{References}

1. Salomão MQ and Wilson SE: Femtosecond laser in laser in situ keratomileusis. J Cataract Refract Surg 36: 1024-1032, 2010.

2. Moshirfar M, Hatch BB, Chang JC, Kurz CJ, Eugarrios MF and Mifflin MD: Prospective, contralateral comparison of $120-\mu \mathrm{m}$ and $90-\mu \mathrm{m}$ LASIK flaps using the IntraLase FS60 femtosecond laser. J Refract Surg 27: 251-259, 2011.

3. Sutton G, Bali SJ and Hodge C: Femtosecond cataract surgery: Transitioning to laser cataract. Curr Opin Ophthalmol 24: 3-8, 2013.

4. He L, Sheehy K and Culbertson W: Femtosecond laser-assisted cataract surgery. Curr Opin Ophthalmol 22: 43-52, 2011.

5. Sutu C, Fukuoka H and Afshari NA: Mechanisms and management of dry eye in cataract surgery patients. Curr Opin Ophthalmol 27: 24-30, 2016.

6. Kasetsuwan N, Satitpitakul V, Changul T and Jariyakosol S: Incidence and pattern of dry eye after cataract surgery. PLoS One 8: e78657, 2013

7. Cho YK and Kim MS: Dry eye after cataract surgery and associated intraoperative risk factors. Korean J Ophthalmol 23: 65-73, 2009.

8. Homma M, Tojo T, Akizuki M and Yamagata H: Criteria for Sjögren's syndrome in Japan. Scand J Rheumatol (Suppl) 61: 26-27, 1986.

9. Ozcura F, Aydin S and Helvaci MR: Ocular surface disease index for the diagnosis of dry eye syndrome. Ocul Immunol Inflamm 15: 389-393, 2007.
10. Jiang Y, Ye H, Xu J and Lu Y: Noninvasive keratograph assessment of tear film break-up time and location in patients with age-related cataracts and dry eye syndrome. J Int Med Res 42: 494-502, 2014.

11. Hong J, Sun X, Wei A, Cui X, Li Y, Qian T, Wang W and $\mathrm{Xu} \mathrm{J}$ : Assessment of tear film stability in dry eye with a newly developed keratograph. Cornea 32: 716-721, 2013.

12. Yu EY, Leung A, Rao S and Lam DS: Effect of laser in situ keratomileusis on tear stability. Ophthalmology 107: 2131-2135, 2000.

13. Albietz JM, Lenton LM and McLennan SG: Effect of laser in situ keratomileusis for hyperopia on tear film and ocular surface. J Refract Surg 18: 113-123, 2002.

14. Müller LJ, Vrensen GF, Pels L, Cardozo BN and Willekens B: Architecture of human corneal nerves. Invest Ophthalmol Vis Sci 38: 985-994, 1997.

15. Pisella PJ, Pouliquen P and Baudouin C: Prevalence of ocular symptoms and signs with preserved and preservative free glaucoma medication. Br J Ophthalmol 86: 418-423, 2002.

16. Herreras JM, Pastor JC, Calonge M and Asensio VM: Ocular surface alteration after long-term treatment with an antiglaucomatous drug. Ophthalmology 99: 1082-1088, 1992.

17. Cetinkaya S, Mestan E, Acir NO, Cetinkaya YF, Dadaci Z and Yener HI: The course of dry eye after phacoemulsification surgery. BMC Ophthalmol 15: 68, 2015.

18. Yokoi N, Takehisa Y and Kinoshita S: Correlation of tear lipid layer interference patterns with the diagnosis and severity of dry eye. Am J Ophthalmol 122: 818-824, 1996.

19. White KM, Benjamin WJ and Hill RM: Human basic tear fluid osmolarity. II. Importance of processing strategy. Acta Ophthalmol (Copenh) 71: 530-538, 1993.

20. Shaheen BS, Bakir M and Jain S: Corneal nerves in health and disease. Surv Ophthalmol 59: 263-285, 2014.

21. Salomão MQ, Ambrósio R Jr and Wilson SE: Dry eye associated with laser in situ keratomileusis: Mechanical microkeratome versus femtosecond laser. J Cataract Refract Surg 35: 1756-1760, 2009.

22. Rodriguez-Prats JL, Hamdi IM, Rodriguez AE, Galal A and Alio JL: Effect of suction ring application during LASIK on goblet cell density. J Refract Surg 23: 559-562, 2007. 